International Journal of Pure and Applied Mathematics

Volume 107 No. 1 2016, 35-48

ISSN: 1311-8080 (printed version); ISSN: 1314-3395 (on-line version)

url: http://www.ijpam.eu

doi: 10.12732 /ijpam.v107i1.4

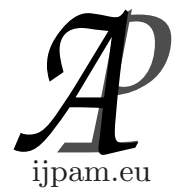

\title{
VAGUE PRIME LI-IDEALS OF LATTICE IMPLICATION ALGEBRAS
}

\author{
V. Amarendra Babu ${ }^{1}$, T. Anitha ${ }^{2} \S$ \\ ${ }^{1}$ Department of Mathematics \\ Achraya Nagarjuna University \\ Nagarjuna Nagar, 522510, INDIA \\ ${ }^{2}$ K.L. University \\ Vaddeswaram, Guntur 522 502, A.P, INDIA
}

\begin{abstract}
In this paper,we study the notion of vague prime LI - ideals of lattice implication algebras. We provide the equivalent conditions for vague prime LI - ideals. Extension property of a vague prime LI - ideal is built. We study the relations between vague prime LI - ideals and Vague ultra LI - ideals, VILI - ideals and Vague ultra LI - ideals.
\end{abstract}

AMS Subject Classification: 03G10, 08A72, 03E72

Key Words: lattice implication algebras, VLI - ideals, VILI - ideals, vague prime LI - ideals, vague ultra $L I$ - ideals

\section{Introduction}

In order to investigate a many - valued logical system whose proportional value is given in a lattice, in $1993 \mathrm{Y}$. Xu [12] first established the lattice implication algebra by combining lattice and implication algebra, and explored many useful structures. The ideal theory serves a vital function for the development of lattice implication algebras. Y. Xu, Y.B. Jun and E.H. Roh [6] introduced the notion of LI - ideals of a lattice implication algebras. In particular, Y.B. Jun

Received: January 10, 2016

Published: March 23, 2016

${ }^{\S}$ Correspondence author (c) 2016 Academic Publications, Ltd.

url: www.acadpubl.eu 
[5] introduced the concept of prime LI - ideals of lattice implication algebras and discussed some of their properties. Later Y. Lin Liu, San Yang Liu, Y. Xu, Ke Yun Qin [13] introduced the notion of ILI - ideals of a lattice implication algebras and obtained relations between prime LI - ideals and LI -ideals, prime LI - ideals and ILI -ideals etc. Ke Yun Qin, Y. Xu and Y.B. Jun [9] introduced the notion of Ultra LI - ideals in lattice implication algebras.

The concept of fuzzy set was introduced by Zadeh [14]. With the development of fuzzy set, it is widely used in many fields. A fuzzy set is a single function, it cannot express the evidence of supporting and opposing. The concept of vague set introduced by W.L. Gau and D.J. Buehrer [4] in 1993. The idea of vague set is that the membership of every element can be divided into two aspects including supporting and opposing. Ranjit Biswas [3] initiated the study of vague algebra by studying vague groups. At first Ya Qin and Yi Liu [10] applied the concept of vague set theory to lattice implication algebras and introduced the notion of $\mathrm{v}$ - filter, and investigated their some properties.

In this paper, we introduce the notion of vague Prime LI - ideals of a lattice implication algebras and discuss some of their properties. Extension property of a vague prime LI - ideal is built. We study the relations between vague prime LI - ideals and Vague ultra LI - ideals, VILI - ideals and Vague ultra LI - ideals.

Throughout this article, $L$ denote a lattice implication algebra.

\section{Preliminaries}

Definition 1. (see [11]) Let $(L, \vee, \wedge, 0, I)$ be a complemented lattice with the universal bounds $0, I . \rightarrow$ is another binary operation of $L$. In the sequel the binary operation $\rightarrow$ will be denoted by juxtaposition. $\left(L, \vee, \wedge, \rightarrow,{ }^{\prime}, 0, I\right)$ is called a lattice implication algebra, if the following axioms hold, $\forall x, y, z \in L$, $\left(I_{1}\right) x(y z)=y(x z)$

$\left(I_{2}\right) x x=I$

(I $\left.I_{3}\right) x y=y^{\prime} x^{\prime}$

$\left(I_{4}\right) x y=y x=I$ implies $x=y$;

$\left(I_{5}\right)(x y) y=(y x) x$

$\left(L_{1}\right)(x \vee y) z=(x z) \wedge(y z)$

$\left(L_{2}\right)(x \wedge y) z=(x z) \vee(y z)$.

A lattice implication algebra $\left(L, \vee, \wedge, \rightarrow,,^{\prime} 0, I\right)$ is called a lattice $H$ implication algebra, if $x \vee y \vee((x \wedge y) z)=I, \forall x, y, z \in L$.

Theorem 2. (see [12]) Let $L$ be a lattice implication algebra, then for any 
$x, y, z \in L$, the following conclusions hold:

1. If $I x=I$ then $x=I$.

2. $I x=x$ and $x 0=x$.

3. $0 x=I$ and $x I=I$.

4. $x \leq y$ if and only if $x y=I$.

5. $(x y) \vee(y x)=I$

6. $x y \geq x^{\prime} \vee y$.

Definition 3. (see [6]) Let $I$ be a nonempty subset of $L$. I is said to be a $L I$ - ideal of $L$ if it satisfies the following conditions:

1. $0 \in I$

2. $\forall x, y \in L,(x y)^{\prime} \in I$ and $y \in I$ imply $x \in I$.

Definition 4. (see [5]) A proper LI - ideal $P$ of $L$ is said to be prime LI ideal if whenever $x \wedge y \in P$ then $x \in P$ or $y \in P$ for all $x, y \in L$.

Definition 5. (see [9]) An LI - ideal $A$ of $L$ is said to be an ultra if for every $x \in L$, the equivalence holds: $x \in A \Leftrightarrow x^{\prime} \notin A$.

Definition 6. (see [4]) $A$ vague set $A$ in the universal of discourse $X$ is characterized by two membership functions given by:

1. A truth membership function $t_{A}: X \rightarrow[0,1]$ and

2. A false membership function $f_{A}: X \rightarrow[0,1]$.

Here $t_{A}$ is a lower bound of the grade of membership of $x$ derived from the 'evidence for $x^{6}$, and $f_{A}$ is a lower bound on the negation of $x$ derived from the "evidence against $x^{6}$ and $t_{A}+f_{A} \leq 1$. Thus the grade of membership of $x$ in the vague set $A$ is bounded by subinterval $\left[t_{A}, 1-f_{A}\right]$ of $[0,1]$. The vague set $A$ is written as $A=\left\{\left\langle x,\left[t_{A}, f_{A}\right]\right\rangle / x \in X\right\}$. Where the interval $\left[t_{A}, 1-f_{A}\right]$ is called the vague value of $x$ in the vague set $A$ and denoted by $V_{A}(x)$.

Definition 7. (see [4]) The $\alpha$ - cut, $A_{\alpha}$ of the vague set $A$ is the $(\alpha, \alpha)$ cut of $A$ and hence given by $A_{\alpha}=\left\{x \in X / t_{A}(x) \geq \alpha\right\}$

Definition 8. (see [1]) Let $A$ be a vague set of a lattice implication algebra $L$. $A$ is said to be a vague LI - ideal (brieflyVLI - ideal) of $L$ if it satisfies the following conditions: 
1. $\forall x \in L, V_{A}(0) \geq V_{A}(x)$;

2. $\forall x, y \in L, V_{A}(x) \geq \operatorname{imin}\left\{V_{A}\left((x y)^{\prime}\right), V_{A}(y)\right\}$.

Definition 9. (see [2]) Let $A$ be a vague set set of a lattice implication algebra $L . A$ is said to be a vague implicative LI - ideal (brieflyVILI - ideal) of $L$ if it satisfies the following conditions:

1. $\forall x \in L, V_{A}(0) \geq V_{A}(x)$;

2. $\forall x, y, z \in L, V_{A}\left((x y)^{\prime}\right) \geq \operatorname{imin}\left\{V_{A}\left(\left(\left((x y)^{\prime} y\right)^{\prime} z\right)^{\prime}\right), V_{A}(z)\right\}$.

\section{Vague Prime Li - Ideals}

In this section, we introduce the notion of Vague prime LI - ideals and investigate some of their properties. We study the relations between vague prime LI - ideals and Vague ultra LI - ideals, VILI - ideals and Vague ultra LI - ideals.

Definition 10. A vague $L I$ - ideal $A$ of $L$ is said to be a vague prime $L I$ ideal if it is a non- constant and $V_{A}(x \wedge y)=V_{A}(x) \vee V_{A}(y)$ for any $x, y \in L$.

Example 11. (see [12]) Let $L=\{0, a, b, c, d, I\}$ be a set with cayley table as follows:

\begin{tabular}{|c|c|c|c|c|c|c|}
\hline$\rightarrow$ & 0 & $a$ & $b$ & $c$ & $d$ & $I$ \\
\hline 0 & $I$ & $I$ & $I$ & $I$ & $I$ & $I$ \\
\hline$a$ & $c$ & $I$ & $b$ & $c$ & $b$ & $I$ \\
\hline$b$ & $d$ & $a$ & $I$ & $b$ & $a$ & $I$ \\
\hline$c$ & $a$ & $a$ & $I$ & $I$ & $a$ & $I$ \\
\hline$d$ & $b$ & $I$ & $I$ & $b$ & $I$ & $I$ \\
\hline$I$ & 0 & $a$ & $b$ & $c$ & $d$ & $I$ \\
\hline
\end{tabular}

Define $^{\prime}, \vee, \wedge$ operations on $L$ as follows:

$x^{\prime}=x \rightarrow 0, x \vee y=(x \rightarrow y) \rightarrow y, x \vee y=\left(\left(x^{\prime} \rightarrow y^{\prime}\right) \rightarrow y^{\prime}\right)^{\prime}$ for all $x, y \in L$.

Then $\left(L, \vee, \wedge, \rightarrow,^{\prime}, 0, I\right)$ is a lattice implication algebra. Let $A$ be a vague set of $L$ defined by 


$$
\begin{aligned}
A=\{\langle 0,[0.6,0.3]\rangle,\langle a,[0.6,0.3]\rangle,\langle b,[0.5,0.4]\rangle, & \langle c,[0.5,0.4]\rangle, \\
& \langle d,[0.6,0.3]\rangle,\langle I,[0.5,0.4]\rangle\} .
\end{aligned}
$$

Then $A$ is a vague prime $L I$ - ideal of $L$.

Lemma 12. Let $A$ be a vague $L I$ - ideal of $L$. Then $A$ is a constant vague set if and only if $V_{A}(0)=V_{A}(I)$

Proof. Necessariness is obvious and we need to prove the sufficiency:

Assume that $A$ satisfies $V_{A}(0)=V_{A}(I)$. Since $A$ is a vague LI - ideal, so $A$ is order reversing. For any $x \in L, 0 \leq x \leq I$, it follows that $V_{A}(0) \geq V_{A}(x) \geq$ $V_{A}(I)$. Hence $V_{A}(0)=V_{A}(I)=V_{A}(x)$ for any $x \in L$. Then $A$ is constant.

Theorem 13. Let $A$ be a non - constant vague $L I$ - ideal of $L$. Then the following are equivalent:

1. $A$ is a vague prime $L I$ - ideal.

2. $\forall x, y \in L$, if $V_{A}(x \wedge y)=V_{A}(0)$, then $V_{A}(x)=V_{A}(0)$ or $V_{A}(y)=V_{A}(0)$.

3. $\forall x, y \in L, V_{A}\left((x y)^{\prime}\right)=V_{A}(0)$ or $V_{A}\left((y x)^{\prime}\right)=V_{A}(0)$.

Proof. (1) $\Rightarrow(2)$

Assume that $A$ is vague prime LI - ideal of $L$.

$$
\begin{array}{cll}
\text { Let } \quad x, y \in L \quad \text { suchthat } & & V_{A}(x \wedge y)=V_{A}(0) \\
\Rightarrow & V_{A}(x) \vee V_{A}(y)=V_{A}(0) \\
\Rightarrow & V_{A}(x)=V_{A}(0) \operatorname{or} V_{A}(y)=V_{A}(0) .
\end{array}
$$

$(2) \Rightarrow(3)$

$$
\begin{aligned}
& \text { Let } \quad x, y \in L \text { then }(x y)^{\prime} \wedge(y x)^{\prime}=0 \\
& \Rightarrow \quad V_{A}\left((x y)^{\prime} \wedge(y x)^{\prime}\right)=V_{A}(0) \\
& \Rightarrow \quad V_{A}\left((x y)^{\prime}\right)=V_{A}(0) \text { or } V_{A}\left((y x)^{\prime}\right)=V_{A}(0) \text {. }
\end{aligned}
$$

$(3) \Rightarrow(1)$ 
Let $x, y \in L$. Suppose that, $V_{A}\left((x y)^{\prime}\right)=V_{A}(0)$. Since $A$ is Vague LI ideal of $L$, it follows that

$$
\begin{aligned}
V_{A}(x) & \geq \operatorname{imin}\left\{V_{A}\left(\left(x(x y)^{\prime}\right)^{\prime}\right), V_{A}\left((x y)^{\prime}\right)\right\} \\
& =\operatorname{imin}\left\{V_{A}\left(\left((x y) x^{\prime}\right)^{\prime}\right), V_{A}(0)\right\} \\
& =\operatorname{imin}\left\{V_{A}(x \wedge y), V_{A}(0)\right\} \\
& \geq V_{A}(x \wedge y) .
\end{aligned}
$$

Since $x \geq x \wedge y, V_{A}(x) \leq V_{A}(x \wedge y)$. Therefore $V_{A}(x \wedge y)=V_{A}(x)$.

Similarly, $V_{A}(x \wedge y)=V_{A}(y)$. Thus $V_{A}(x \wedge y)=V_{A}(x) \vee V_{A}(y)$.

Simillarly we can prove that, $V_{A}\left((y x)^{\prime}\right)=V_{A}(0)$ implies $V_{A}(x \wedge y)=V_{A}(x) \vee V_{A}(y)$.

Therefore, $A$ is a vague prime LI - ideal of $L$.

Corollary 14. Let $P$ be a vague prime $L I$ - ideal of $L$ then every non constant VLI - ideal containing $P$ is also vague prime $L I$ - ideal of $L$.

Corollary 15. Let $A$ be a vague prime $L I$ - ideal of $L$ then the $\alpha$-cut, $A_{\alpha}$ is also vague prime $L I$ - ideal of $L$.

Theorem 16. Let $A$ be a vague $L I$ - ideal of $L$. Then $A$ is a vague prime $L I$ - ideal if and only if the set $I=\left\{x \in L / V_{A}(x)=V_{A}(0)\right\}$ is a prime $L I$ ideal.

Proof. Suppose that $A$ is a vague prime LI - ideal $L$.

Since $A$ is non - constant, $I$ is proper.

$$
\begin{array}{cll}
\text { Let } \quad x, y \in L \quad \text { suchthat } & x \wedge y \in I \\
\Rightarrow & V_{A}(0)=V_{A}(x \wedge y)=V_{A}(x) \vee V_{A}(y) \\
\Rightarrow & V_{A}(x)=V_{A}(0) \text { or } V_{A}(y)=V_{A}(0) \\
\Rightarrow & x \in I \text { or } y \in I .
\end{array}
$$

Therefore, $I$ is a prime LI - ideal of $L$.

Conversely, assume that $I$ is a prime LI - ideal of $L$.

Since $I$ is proper, $A$ is non - constant.

$$
\text { Let } \begin{aligned}
& x, y \in L \quad \text { then }(x y)^{\prime} \wedge(y x)^{\prime}=0 \\
& \Rightarrow \quad(x y)^{\prime} \in I \quad \text { or } \quad(y x)^{\prime} \in I
\end{aligned}
$$




$$
\Rightarrow \quad V_{A}(x y)^{\prime}=V_{A}(0) \quad \text { or } \quad V_{A}(y x)^{\prime}=V_{A}(0)
$$

So $A$ is a vague prime LI - ideal $L$.

Example 17. In example 11, the vague set $A$ is a vague prime LI - ideal of $L$. Then $I=\{0, a, d\}$ is a prime $L I$ - ideal of $L$.

Let $I$ be a subset of of $L$ and $\alpha \in[0,1]$. Now we define vague set $A_{I}$ by

$$
V_{A_{I}}(x)=\left\{\begin{array}{l}
{[\alpha, \alpha] \quad \text { if } \quad x \in I} \\
{[0,0] \quad \text { otherwise }}
\end{array}\right.
$$

Theorem 18. Let $I$ be a VLI - ideal of $L$. Then $I$ is a prime LI - ideal of $L$ if and only if $A_{I}$ is a vague prime $L I$ - ideal of $L$.

Proof. Assume that $I$ is a prime LI - ideal of $L$.

Since $I$ is proper, $A_{I}$ is non - constant.

$$
\begin{aligned}
\text { Let } x, y \in L \quad \text { then } & (x y)^{\prime} \wedge(y x)^{\prime}=0 \\
\Rightarrow \quad & (x y)^{\prime} \in I \quad \text { or } \quad(y x)^{\prime} \in I \\
\Rightarrow \quad & V_{A_{I}}\left((x y)^{\prime}\right)=[\alpha, \alpha]=V_{A_{I}}(0)
\end{aligned}
$$

or

$$
V_{A_{I}}\left((y x)^{\prime}\right)=[\alpha, \alpha]=V_{A_{I}}(0) .
$$

Therefore $A_{I}$ is a vague prime LI - ideal of $L$.

Conversely, assume that $A_{I}$ is a vague prime LI - ideal of $L$.

$$
\begin{array}{cl}
\text { Then } & V_{A_{I}}\left((x y)^{\prime}\right)=V_{A_{I}}(0) \quad \text { or } \quad V_{A_{I}}\left((y x)^{\prime}\right)=V_{A_{I}}(0) \text { forall } x, y \in L \\
\Rightarrow & V_{A_{I}}\left((x y)^{\prime}\right)=[\alpha, \alpha] \quad \text { or } \quad V_{A_{I}}\left((y x)^{\prime}\right)=[\alpha, \alpha] \\
\Rightarrow & (x y)^{\prime} \in I \quad \text { or } \quad(y x)^{\prime} \in I .
\end{array}
$$

So $I$ is a prime LI - ideal of $L$.

Theorem 19. Let $A$ be a proper VLI - ideal of $L$. Then $L$ is a chain if and only if $A$ is prime $L I$ - ideal of $L$.

Proof. Assume that $L$ is a chain and $A$ be any proper VLI - ideal of $L$.

$$
\begin{gathered}
\text { Then } \quad x \leq y \quad \text { or } \quad y \leq x \text { forall } x, y \in L \\
\Rightarrow \quad x y=I \quad \text { or } \quad y x=I
\end{gathered}
$$




$$
\Rightarrow \quad V_{A}\left((x y)^{\prime}\right)=V_{A}(0) \quad \text { or } \quad V_{A}\left((y x)^{\prime}\right)=V_{A}(0)
$$

Therefore $A$ is vague prime LI - ideal of $L$.

Conversely, suppose that $A$ is vague prime LI - ideal of $L$.

$$
\begin{aligned}
\text { Then } & V_{A}\left((x y)^{\prime}\right)=V_{A}(0) \quad \text { or } \quad V_{A}\left((y x)^{\prime}\right)=V_{A}(0) \text { forall } x, y \in L \\
\Rightarrow & x y=I \quad \text { or } \quad y x=I \\
\Rightarrow & x \leq y \text { or } y \leq x
\end{aligned}
$$

So, $L$ is a chain.

Theorem 20. Let $A$ be a non - constant VLI - ideal of $L$ such that for any VLI - ideals $A_{1}, A_{2}$ of $L, A_{1} \wedge A_{2} \leq A$ implies $A_{1} \leq A$ or $A_{2} \leq A$. Then $A$ is a vague prime $L I$ - ideal of $L$.

Proof. On the contradictory assume that $A$ is not a vague prime LI - ideal of $L$. Then $I=\left\{x \in L / V_{A}(x)=V_{A}(0)\right\}$ is not a prime LI - ideal of $L$ by theorem 16. Let $I_{1}, I_{2}$ be two LI - ideals such that $I=I_{1} \cap I_{2}$. Implies $I \subset I_{1}$ and $I \subset I_{2}$ (otherwise $I=I_{1}$ or $I=I_{2}$, then $I$ is irreducible, that is $I$ is prime). Then there exist $x_{1}, x_{2} \in L$ such that $x_{1} \in I_{1}$ but $x_{1} \notin I, x_{2} \in I_{2}$ but $x_{2} \notin I$. Therefore $V_{A}\left(x_{1}\right)<V_{A}(0)$ and $V_{A}\left(x_{2}\right)<V_{A}(0)$. We define two vague sets $A_{1}, A_{2}$ as follows:

$$
\begin{aligned}
& V_{A_{1}}(x)=\left\{\begin{array}{l}
V_{A}(0) \quad \text { if } \quad x \in I_{1}, \\
0 \quad \text { if } \quad x \notin I_{1},
\end{array}\right. \\
& V_{A_{2}}(x)=\left\{\begin{array}{l}
V_{A}(0) \quad \text { if } \quad x \in I_{2}, \\
0 \quad \text { if } \quad x \notin I_{2} .
\end{array}\right.
\end{aligned}
$$

Now, we need to prove $A_{1}, A_{2}$ are VLI - ideals of $L$, but we only to prove $A_{1}$.

Obviously, $V_{A_{1}}(0) \geq V_{A_{1}}(x)$ for any $x \in L$. Let $x, y \in L$

(a) If $y \in I_{1}$ and $(x y)^{\prime} \in I_{1}$, then $x \in I_{1}$. By definition of $A_{1}$, it follows that $V_{A_{1}}(y)=V_{A}(0)=V_{A_{1}}\left((x y)^{\prime}\right)$ and $V_{A_{1}}(x)=V_{A}(0)$.

Therefore $V_{A_{1}}(x) \geq \operatorname{imin}\left\{V_{A_{1}}\left((x y)^{\prime}\right), V_{A_{1}}(y)\right\}$.

(b) If $y \notin I_{1}$ or $(x y)^{\prime} \notin I_{1}$, it is easy to verify

$$
V_{A_{1}}(x) \geq \operatorname{imin}\left\{V_{A_{1}}\left((x y)^{\prime}\right), V_{A_{1}}(y)\right\} \text {. }
$$


Therefore, $A_{1}$ is a VLI - ideal of $L$ by (a) and (b). Similarly we can prove $A_{2}$ is a VLI - ideal of $L$.

For any $x \in L$, it is to verify that $\left(A_{1} \wedge A_{2}\right) \leq A$. By hypothesis, $A_{1} \leq A$ and $A_{2} \leq A$. But, since $V_{A_{1}}\left(x_{1}\right)=V_{A}(0)>V_{A}(x)$ and $V_{A_{2}}\left(x_{2}\right)=V_{A}(0)>$ $V_{A}(x)$, it follows that $A_{1}>A$ and $A_{2}>A$. Contradiction. Thus, $A$ is a vague prime LI - ideal of $L$.

Theorem 21. Let $L_{1}$ and $L_{2}$ are two lattice implication algebras. The mapping $f: L_{1} \rightarrow L_{2}$ is an onto lattice implication homomorphism. Then:

(a) If $B$ is a vague prime $L I$ - ideal of $L_{2}$ then $f^{-1}(B)$ is a vague prime $L I$ - ideal of $L_{1}$.

(b) If $A$ is a vague prime $L I$ - ideal of $L_{1}$ then $f(A)$ is a vague prime $L I$ ideal of $L_{2}$.

Proof. (a) Let $B$ is a vague prime LI - ideal of $L_{2}$. By theorem 3.19 in [1], $f^{-1}(B)$ is a vague LI - ideal of $L_{1}$. Let $x, y \in L_{1}$.

$$
\begin{aligned}
\text { Then } f^{-1}\left(V_{B}(x)\right) \vee f^{-1}\left(V_{B}(y)\right) & =V_{B}(f(x)) \vee V_{B}(f(x)) \\
& =V_{B}(f(x) \wedge f(y)) \\
& =V_{B}(f(x \wedge y)) \\
& =f^{-1}\left(V_{B}(x \wedge y)\right) .
\end{aligned}
$$

So $f^{-1}(B)$ is a vague prime LI - ideal of $L_{1}$.

(b) Let $A$ is a vague prime LI - ideal of $L_{1}$. By theorem 3.19 in [1], $f(A)$ is a vague LI - ideal of $L_{2}$. Since $f: L_{1} \rightarrow L_{2}$ is an onto then for any $x, y \in L_{2}$ there exist $u, v \in L_{1}$ such that $f(u)=x, f(v)=y$.

$$
\begin{aligned}
\text { Then } f\left(V_{A}(x)\right) \vee f\left(V_{A}(y)\right) & =V_{A}\left(f^{-1}(x)\right) \vee V_{A}\left(f^{-1}(y)\right) \\
& =V_{A}(u) \vee V_{A}(v) \\
& =V_{A}(u \wedge v) \\
& =V_{A}\left(f^{-1}(x) \wedge f^{-1}(y)\right) \\
& =V_{A}\left(f^{-1}(x \wedge y)\right) \\
& =f\left(V_{A}(x \wedge y)\right) .
\end{aligned}
$$

So $f(A)$ is a vague prime LI - ideal of $L_{2}$.

Let $A$ be a vague set of a lattice implication algebra $\mathrm{L}$ such that $t_{A}(x)+$ $f_{A}(x) \leq t_{A}(0)+f_{A}(0)$ for all $x \in L$. Define $A^{*}$ such that $V_{A^{*}}(x)=V_{A}(x)+1-$ $V_{A}(0)$, for all $x \in L$, then $A^{*}$ is a vague set of $L$. 
Theorem 22. Let $A$ be a vague set of $L$. Then $A$ is a vague prime $L I$ ideal of $L$ if and only if the vague set $A^{*}$ is vague prime $L I$ - ideal of $L$.

Proof. Proof: Let $A$ be a vague prime LI - ideal of a lattice implication algebra $L$, then $V_{A}(0) \geq V_{A}(x)$ for all $x \in L$. For any $x, y \in L$, $V_{A^{*}}(0)=V_{A}(0)+1-V_{A}(0)=1 \geq V_{A^{*}}(x)$ and

$$
\begin{aligned}
\operatorname{imin}\left\{V_{A^{*}}(y), V_{A^{*}}\left((x y)^{\prime}\right)\right\} & =\operatorname{imin}\left\{V_{A}(y)+1-V_{A}(0), V_{A}\left((x y)^{\prime}\right)+1-V_{A}(0)\right\} \\
& =\operatorname{imin}\left\{V_{A}(y), V_{A}\left((x y)^{\prime}\right)\right\}+1-V_{A}(0) \\
& \leq V_{A}(x)+1-V_{A}(0) \\
& =V_{A^{*}}(x) .
\end{aligned}
$$

Therefore $A^{*}$ is a vague LI - ideal of $L$. Now, we prove $A^{*}$ is prime.

Since $A$ is vague prime LI - ideal, for any $x, y \in L$,

$$
\begin{aligned}
V_{A}(x \wedge y) & =V_{A}(x) \vee V_{A}(y) \\
V_{A}(x \wedge y)+1-V_{A}(0) & =\left(V_{A}(x) \vee V_{A}(y)\right)+1-V_{A}(0) \\
V_{A}(x \wedge y)+1-V_{A}(0) & =\left(V_{A}(x)+1-V_{A}(0)\right) \vee\left(V_{A}(y)+1-V_{A}(0)\right) \\
V_{A^{*}}(x \wedge y) & =V_{A^{*}}(x) \vee V_{A^{*}}(y),
\end{aligned}
$$

so $A^{*}$ is a vague prime LI - ideal of $L$.

Conversely, suppose that $A^{*}$ is a vague prime LI - ideal, then $V_{A^{*}}(x) \leq$ $V_{A^{*}}(0)$, that is $V_{A}(x)+1-V_{A}(0) \leq V_{A}(0)+1-V_{A}(0)$, it follows that $V_{A}(x) \leq$ $V_{A}(0)$. $V_{A}(x)$.

Since $\min \left\{V_{A^{*}}(y), V_{A^{*}}\left((x y)^{\prime}\right)\right\} \leq V_{A^{*}}(x)$, so $\min \left\{V_{A}(y), V_{A}\left((x y)^{\prime}\right)\right\} \leq$

As $A^{*}$ is prime, it follows that $V_{A^{*}}(x \wedge y)=V_{A^{*}}(x) \vee V_{A^{*}}(y)$, we have $V_{A}(x \wedge y)=V_{A}(x) \vee V_{A}(y)$. Therefore, $A$ is a vague prime LI - ideal of $L$.

Definition 23. A vague $L I$ - ideal of $L$ is said to be a vague ultra $L I$ ideal of $L$ if for any $x \in L$, either $V_{A}(x)=V_{A}(0)$ or $V_{A}\left(x^{\prime}\right)=V_{A}(0)$.

Example 24. Clearly the vague set $A$ in the example 11 of $L$ is a vague ultra $L I$ - ideal if $L$.

Theorem 25. Every non constant vague ultra $L I$ - ideal of $L$ is a vague prime $L I$ - ideal of $L$. 
Proof. Suppose that $A$ is a vague ultra LI - ideal of $L$.

Then for any $x \in L$, either $V_{A}(x)=V_{A}(0)$ or $V_{A}\left(x^{\prime}\right)=V_{A}(0)$.

Let us consider for any $x, y \in L, V_{A}(x \wedge y)=V_{A}(0)$.

If $V_{A}(x)=V_{A}(0)$ then obviously, $A$ is a vague prime LI - ideal.

If $V_{A}\left(x^{\prime}\right)=V_{A}(0)$. For any $x, y \in L$.

As $\left(y x^{\prime}\right)^{\prime} \leq\left(y^{\prime} \vee x^{\prime}\right)^{\prime}=y \wedge x$ and $A$ is a VLI - ideal, it follows that

$$
\begin{aligned}
V_{A}(y) & \geq \operatorname{imin}\left\{V_{A}\left(\left(y x^{\prime}\right)^{\prime}\right), V_{A}\left(x^{\prime}\right)\right\} \\
& \geq \operatorname{imin}\left\{V_{A}(y \wedge x), V_{A}\left(x^{\prime}\right)\right\} \\
& \geq \operatorname{imin}\left\{V_{A}(0), V_{A}(0)\right\} \\
& =V_{A}(0)
\end{aligned}
$$

and $V_{A}(y) \leq 0$. Thus $V_{A}(y)=V_{A}(0)$. By theorem $13 A$ is a vague prime LI ideal.

Theorem 26. Every vague ultra $L I$ - ideal of $L$ is a VILI -ideal of $L$.

Proof. Suppose that $A$ is a vague ultra LI - ideal of $L$. Since $A$ is a vague prime LI - ideal, $V_{A}\left((x y)^{\prime}\right)=V_{A}(0)$ or $V_{A}\left((y x)^{\prime}\right)=V_{A}(0)$ for all $x, y \in L$.

For instance $V_{A}\left((x y)^{\prime}\right)=V_{A}(0)$, since $A$ is a VLI - ideal of $L, V_{A}(0) \geq$ $V_{A}(x)$ for all $x \in L$.

$$
\begin{aligned}
\text { Itfollowsthat } V_{A}\left((x y)^{\prime}\right) & =V_{A}(0) \\
& \geq V_{A}\left(\left((x y)^{\prime} y\right)^{\prime}\right) \\
& \geq \operatorname{imin}\left\{V_{A}\left(\left(\left((x y)^{\prime} y\right)^{\prime} z\right)^{\prime}\right), V_{A}(z)\right\} .
\end{aligned}
$$

Similarly, If $V_{A}\left((y x)^{\prime}\right)=V_{A}(0)$, then we obtain

$$
V_{A}\left((y x)^{\prime}\right) \geq \operatorname{imin}\left\{V_{A}\left(\left(\left((y x)^{\prime} x\right)^{\prime} z\right)^{\prime}\right), V_{A}(z)\right\} .
$$

Hence $A$ is a VILI - ideal of $L$. 
Theorem 27. In a Lattice $H$ implication algebra, every vague prime $L I$ ideal is a vague ultra LI - ideal.

Proof. Let $A$ be a vague prime LI - ideal of a Lattice $\mathrm{H}$ implication algebra $L$. Then for any $x \in L$, we have $x \vee x^{\prime}=I$. So, $V_{A}\left(x^{\prime} \wedge x\right)=V_{A}(0)$. By theorem 13 , we have $V_{A}(x)=V_{A}(0)$ or $V_{A}\left(x^{\prime}\right)=V_{A}(0)$. Hence $A$ is a vague ultra LI - ideal of $L$.

Remark 28. In a Lattice $H$ implication algebra, the concepts of vague prime $L I$ - ideals and vague ultra $L I$ - ideals coincide.

Theorem 29. Let $A$ be a proper vague $L I$ - ideal of $L$. Then $A$ is vague ultra $L I$ - ideal of $L$ if and only if $V_{A}(x \otimes y)=V_{A}(0)$ implies $V_{A}(x)=V_{A}(0)$ or $V_{A}(y)=V_{A}(0)$ for any $x, y \in L$.

Proof. Suppose that $A$ is a vague ultra LI - ideal of $L$ and $V_{A}(x \otimes y)=$ $V_{A}(0)$. So $V_{A}\left(x^{\prime} \oplus y^{\prime}\right)=V_{A}\left(x y^{\prime}\right)=V_{A}\left((x \otimes y)^{\prime}\right) \neq V_{A}(0)$.

Since $A$ is a vague LI - ideal, $V_{A}\left(x^{\prime} \oplus y^{\prime}\right) \geq \operatorname{imin}\left\{V_{A}\left(x^{\prime}\right), V_{A}\left(y^{\prime}\right)\right\}$.

It follows that $V_{A}\left(x^{\prime}\right) \neq V_{A}(0)$ or $V_{A}\left(y^{\prime}\right) \neq V_{A}(0)$.

That is $V_{A}(x)=V_{A}(0)$ or $V_{A}(y)=V_{A}(0)$.

Conversely, suppose that is a for any $x, y \in L, V_{A}(x \otimes y)=V_{A}(0)$ implies $V_{A}(x)=V_{A}(0)$ or $V_{A}(y)=V_{A}(0)$.

For any $x \in L$, we have $V_{A}\left(x \otimes x^{\prime}\right)=V_{A}\left((x x)^{\prime}\right)=V_{A}(0)$, which implies $V_{A}(x)=V_{A}(0)$ or $V_{A}\left(x^{\prime}\right)=V_{A}(0)$.

So $A$ is a vague ultra LI - ideal of $L$.

Theorem 30. Let $L_{1}$ and $L_{2}$ are two lattice implication algebras. The mapping $f: L_{1} \rightarrow L_{2}$ is an onto lattice implication homomorphism and $f(0)=$ 0. Then:

(a) If $B$ is a vague ultra $L I$ - ideal of $L_{2}$ then $f^{-1}(B)$ is a vague ultra $L I$ ideal of $L_{1}$.

(b) If $A$ is a vague ultra $L I$ - ideal of $L_{1}$ and $f$ is bijective then $f(A)$ is a vague ultra $L I$ - ideal of $L_{2}$.

Proof. (a) Let $B$ is a vague ultra LI - ideal of $L_{2}$.

By theorem 3.19 in [1], $f^{-1}(B)$ is a vague LI - ideal of $L_{1}$.

Let $x, y \in L_{1}$, then $V_{B}(f(x))=V_{B}(f(0))=V_{B}(0)$ or $V_{B}\left(f\left(x^{\prime}\right)\right)=$ $V_{B}(f(0))=V_{B}(0)$. 
That is $f^{-1}\left(V_{B}(x)\right)=f^{-1}\left(V_{B}(0)\right)$ or $f^{-1}\left(V_{B}\left(x^{\prime}\right)\right)=f^{-1}\left(V_{B}(0)\right)$.

So $f^{-1}(B)$ is a vague ultra LI - ideal of $L_{1}$.

(b) Let $A$ is a vague ultra LI - ideal of $L_{1}$.

By theorem 3.19 in [1], $f(A)$ is a vague LI - ideal of $L_{2}$.

Let $f: L_{1} \rightarrow L_{2}$ is an bijective. $v$.

Let us consider $u, v \in L_{2}$ there exist $x, x^{\prime} \in L_{1}$ such that $f(x)=u, f\left(x^{\prime}\right)=$

Since $A$ is a vague ultra LI - ideal of $L_{1}$, we have

$$
V_{A}(x)=V_{A}(0) \text { or } V_{A}\left(x^{\prime}\right)=V_{A}(0) \text {. }
$$

If $V_{A}(x)=V_{A}(0)$, then $f\left(V_{A}(u)\right)=V_{A} f^{-1}((u))=V_{A}(x)=V_{A}(0)$.

If $V_{A}\left(x^{\prime}\right)=V_{A}(0)$, then $f\left(V_{A}(v)\right)=V_{A} f^{-1}((v))=V_{A}\left(x^{\prime}\right)=V_{A}(0)$.

So $f(A)$ is a vague ultra LI - ideal of $L_{2}$.

\section{Conclusion}

Since W.L.Gau, D.J.Buehrer proposed the notion of vague sets, these ideas have been applied to various fields. In this paper, we applied these ideas to Lattice implication algebras and introduced the notion of vague prime LI ideal. We obtained some properties of vague Prime LI - ideals and established the extension theorem for vague prime LI - ideals. we derive the relations between the vague Prime LI - ideals and vague ultra LI - ideals, VILI - ideals and Vague ultra LI - ideals. it is our sincere with that our work helps in supporting and augmenting various researches in this field.

\section{References}

[1] T. Anitha and V. Amarendra Babu, Vague LI - ideals on lattice implication algebras, J. Emerging Trends in Computing and Information Sciences, 5,(2014), 788 - 793.

[2] V. Amarendra Babu and T. Anitha, Vague implicative LI - ideals on lattice implication algebras, Mathematics and Statistics, 3, No.3(2015,53 - 57.

[3] R. Biswas, vague groups, Int. J. Comput. Congition, 4, No.2(2006), 20 - 23.

[4] W.L. Gau and D.J. Buehrer, Vague sets,IEEE Transactions on Systems, Man and Cybernetics, 23, N0.20(1993), 610-614.

[5] Y.B.Jun, On LI - ideals and prime LI - ideals of lattice implication algebras, J. Korean Math.Soc., 36, (1999), 369 - 380. 
[6] Y.B.Jun, E.H.Roh and Y.Xu, LI - ideals in lattice implication algebras,Bull. Korean Math.Soc., 35, No.1(1998), 13 - 23.

[7] Y.B.Jun and Y.Xu, Fuzzy LI - ideals in lattice implication algebras, J. Fuzzy Math., 7, No.4(1999), 997 - 1003.

[8] Yi Liu, Ke-yun Qin, Yang Xu,Fuzzy prime filters of lattice implication algebras, Fuzzy Inf.Eng., 3, (2011), 235 - 246.

[9] Ke Yun Qin, Y.Xu and Y.B.Jun, Ultra LI - ideals in lattice implication algebras, Czechoslovak mathematical journal, 52, No.3(2002), 463 - 468.

[10] Ya Qin and Yi Liu, V- filter on lattice implication algebras, Journal of Emerging Trends in Computing and Information Sciences, 3, No.9(2012), 1298 - 1301.

[11] Y.Xu, Lattice implication algebras, J. Southwest Jiaotong University, 28, No.1(1993), $20-27$.

[12] Y.Xu, D. Ruan, K.Y.Qin, Lattice - valued Logic, An Alternative Approach to Treat Fuzziness and Incomparability, Springer, (2003).

[13] Y.Xu, Young Lin Liu, San Yang Liu, Ke Yun Qin, ILI - ideals and prime LI - ideals in lattice implication algebras, Information Sciences, 155, (2003), 157 - 175.

[14] L.A. Zadeh, Fuzzy sets, Information and Control, 8, (1965), 338-353. 